\title{
NOTES ON ENERGY FOR SPACE-TIME PROCESSES OVER LÉVY PROCESSES
}

\author{
MAMORU KANDA \\ Dedicated to Professor Masanori Kishi on his 60th birthday
}

\section{§1. Introduction}

Let $X=\left(X_{t}, 0 \leq t<\infty\right)$ be a Lévy process on the Euclidean space $R^{d}$, that is, a process on $R^{d}$ with stationary independent increments which has right continuous paths with left limits. We denote by $P^{x}$ the probability measure such that $P^{x}\left(X_{0}=x\right)=1$ and by $E^{x}$ the expectation relative to $P^{x}$. The process is characterized by the exponent $\Psi$ through

$$
E^{0}\left(\exp i\left\langle z, X_{t}\right\rangle\right)=\exp (-t \Psi(z)) .
$$

The $\lambda$-energy $E_{X}^{\lambda}(\nu)$ of a measure $\nu$ on $R^{d}$ for $X$ is defined by

$$
E_{X}^{\lambda}(\nu)=\int \operatorname{Re}\left([\lambda+\Psi(z)]^{-1}\right)\left|\mathscr{F}_{\nu}(z)\right|^{2} d z,
$$

where $\mathscr{F}$ denotes the Fourier tranform on $R^{d}$. A nice explanation of the reason why it is called the $\lambda$-energy is given in Rao [11]. Throughout the paper $\mathscr{F} \nu(z)$ is defined by $\int \exp i\langle z, x\rangle \nu(d x)$ and we write $\mathscr{F} u(z)$ in place of $\mathscr{F} u d x(z)$ if $\nu(d x)=u(x) d x$. So our $\lambda$-energy differs from Rao's by a constant multiple.

The space-time process $Y=\left(Y_{t}, 0 \leq t<\infty\right)$ over $X$ is a Lévy process on $R^{1} \times R^{d}$ defined on the probability space $\left(R^{1} \times \Omega, P^{r, x}\right)$, where $\Omega$ is the path space of $X$ and $P^{r, x}=\delta_{r} \otimes P^{x}, \delta_{r}$ being the Dirac measure at $r \in R^{1}$. The trajectory $Y_{t}(r, \omega)$ is $\left(r+t, X_{t}(\omega)\right)$ and the exponent of $Y$ is $\Psi(z)-i t$. So the $\lambda$-energy $E_{Y}^{\lambda}(\mu)$ of a measure $\mu$ on $R^{1} \times R^{d}$ for $Y$ is

$$
E_{Y}^{\lambda}(\mu)=\iint \operatorname{Re}\left([\lambda+\Psi(z)-i t]^{-1}\right)|\mathscr{F} \mu(t, z)|^{2} d t d z,
$$

where $\mathscr{F}$ denotes the Fourier transform on $R^{1} \times R^{d}$.

Received January 10, 1990. 
If we assume the existence of a transition probability density $p(t, x)$ of $X$ relative to the Lebesgue measure $d x$, that is, $P^{0}\left(X_{t} \in d x\right)=p(t, x) d x$, the $\lambda$-resolvent density $U^{2}(x)$ of $X$ is $\int_{0}^{\infty} \exp (-\lambda t) p(t, x) d t$ and the $\lambda$ resolvent density $W^{\lambda}(t, x)$ of $Y$ relative to the Lebesgue measure $d t d x$ on $R^{1} \times R^{d}$ is

$$
\exp (-\lambda t) 1_{] 0, \infty[}(t) p(t, x) .
$$

In this paper we show

Theorem. Let $X$ be a Lévy process on $R^{d}$ with a transition probability density, and $Y$ be the space-time process over $X$. Let $\mu$ be a bounded measure on $R^{1} \times R^{d}$ of compact support.

( I) Assume that the ג-energy of $\mu$ for $Y$ is finite. Then we have the following.

(i) The $R^{d}$-marginal $\mu_{2}$ of $\mu$ (i.e. $\mu_{2}(B)=\mu\left(R^{1} \times B\right)$ ) has finite $\lambda$ energy for $X$.

(ii) If the $R^{1}$-marginal $\mu_{1}$ of $\mu$ (i.e. $\mu_{1}(B)=\mu\left(B \times R^{d}\right)$ ) is singular to the Lebesgue measure on $R^{1}$, then the $R^{d}$-marginal $\mu_{2}$ does not charge any semipolar set.

(II) Consider the case that $\mu$ is of the direct product form $\eta \otimes \nu$.

(i) If $\mu$ has finite $\lambda$-energy for $Y$ and $\nu$ is carried by a semipolar set for $X$, then $\eta$ has a $L^{2}$-density relative to the Lebesgue measure on $R^{1}$.

(ii) If $\nu$ is a bounded measure of compact support on $R^{d}$ with finite $\lambda$-energy for $X$ and it does not charge any semipolar set for $X$, then we can find a singular measure $\eta$ of compact support so that $\mu=\eta \otimes \nu$ has finite $\lambda$-energy for $Y$.

Using Theorem, we can get a new characterization of semipolar sets, which is announced for a more general class of Markov processes with transition probability density [9].

Corollary. Let $X$ be a Lévy process on $R^{d}$ which has a transition probability density. Then a closed set $B$ in $R^{d}$ is semipolar if and only if

$$
P^{x}\left(X_{t} \in B \text { for some } t \in A\right)=0
$$

for every $x \in R^{d}$ and every set $\left.A \subset\right] 0, \infty[$ of Lebesgue measure 0 .

Remark. The above Corollary does not hold if we do not assume the existence of a transition probability density. Indeed, let $X$ be the 
space-time Brownian motion on $R^{1} \times R^{d}$ and let $B=\left\{\left(t_{0}, x\right), x \in R^{d}\right\}$. Then $P^{0}\left(X_{t_{0}} \in B\right)=1$, but $B$ is semipolar.

In $\S 2$ we shall prepare some notations and several lemmas. The proof of Theorem and Corollary will be given in the subsequenct sections.

\section{§ 2. Preliminaries}

Throughout this section we assume that the Lévy process $X$ has a $\lambda$-resolvent density $U^{\lambda}(x)$, that is,

$$
\int_{0}^{\infty} \exp (-\lambda t) P^{0}\left(X_{t} \in d x\right) d t=U^{\lambda}(x) d x
$$

But we do not assume the existence of a transition probability density. So all the results in this section hold for the space-time process $Y$ over $X$, if $X$ has a transition probability density. We note that $U^{2}$ is always chosen to be lower semicontinuous. See Hawkes [4]. The convolution operation is written as "*". The symbol " " is used to denote the reflection, that is, $\tilde{\mu}(d y)=\mu(-d y), \tilde{f}(x)=f(-x)$. The symmetrized $\lambda$ resolvent density is written as $U_{S}^{\lambda}$ :

$$
U_{S}^{\lambda}(x)=\left\{U^{\lambda}(x)+U^{2}(-x)\right\} / 2
$$

Then

$$
\mathscr{F}\left(U_{S}^{\lambda}\right)(z)=\operatorname{Re}\left([\lambda+\Psi(z)]^{-1}\right),
$$

where $\Psi$ is the exponent of $X$.

The celebrated theorem of Bochner plays an important role in the proof of Theorem. So we repeat it here:

Let $f$ be bounded in a neighborhood of the origin and belong to $L^{1}$. If $\mathscr{F}(f)$ is nonnegative, then $\mathscr{F}(f)$ belong to $L^{1}$ and $f=\mathscr{F}^{-1}(\mathscr{F}(f))$ almost surely.

Applying this theorem to our case, we have

LEMMA 2.1. The $\lambda$-energy $E_{X}^{\lambda}(\mu)$ of a measure $\mu$ for $X$ is finite if and only if $U_{S}^{\lambda} * \mu * \tilde{\mu}$ is bounded. If $E_{X}^{\lambda}(\mu)$ is finite, then

$$
U_{S}^{\lambda} * \mu * \tilde{\mu}=\mathscr{F}^{-1}\left[\operatorname{Re}\left([\lambda+\Psi]^{-1}\right)|\mathscr{F} \mu|^{2}\right]
$$

almost everywhere, and so

$$
U_{S}^{\lambda} * \mu * \tilde{\mu}(0) \leq(2 \pi)^{-d} E_{X}^{\lambda}(\mu) .
$$


The last inequality follows from the lower semicontinuity of $U_{S}^{\lambda} * \mu * \tilde{\mu}$ and the continuity of the right-hand side of the equality. Using this lemma we can prove

Corollary of Lemma 2.1. If $E_{X}^{\lambda}(\mu)$ is finite, then $E_{X}^{\lambda}(\mu)$ is monotone decreasing as $\lambda$ increases. If $\mu=\mu_{1}+\mu_{2}$, where $\mu_{i}, i=1,2$, are measures. then $E_{X}^{\lambda}(\mu) \geq E_{X}^{\lambda}\left(\mu_{i}\right), i=1,2$.

The first assertion follows from the monotone decreasingness of $U_{S}^{\lambda} * \mu * \tilde{\mu}(x)$ in $\lambda$ for every fixed $x$. The second statement follows from the inequality $U_{S}^{\lambda} * \mu * \tilde{\mu}(x) \geq U_{S}^{\lambda} * \mu_{i} * \tilde{\mu}_{i}(x)$ for every $x$.

Let $C^{\lambda}(K)$ be the $\lambda$-capacity of a Borel set $K$, that is, the total mass of the uniquely determined measure $\pi$ on the closure of $K$ such that $\tilde{U}^{2} * \pi(x)=E^{x}\left(\exp \left(-\lambda T_{K}\right)\right)$, where $T_{K}=\inf \left(t>0, X_{t} \in K\right)$. The following lemma is proved essentially by Kanda [5] and Hawkes [4] without explicit mentioning. The explicit statement (proved from a very different point of view) is given by Rao.

Lemma 2.2 Rao ([11]). Let $K$ be a compact set and $\nu$ be a bounded measure on $K$. Then

$$
E_{X}^{\lambda}(\nu) \geq(2 \pi)^{d}|\nu(K)|^{2} / 2 C^{\lambda}(K) .
$$

We say that a Borel set $B$ is thin if $E^{x}\left(\exp \left(-\lambda T_{B}\right)\right)<1$ for every $x \in R^{d}$. The set $B$ is semipolar if $B$ is a countable union of thin sets. The set $B$ is called polar if $E^{x}\left(\exp \left(-\lambda T_{B}\right)\right)=0$ for every $x$. Then we can give a characterization of polar sets using $\lambda$-energy.

Lemma 2.3 (Kanda [6], Hawkes [4] and Rao [11]). A Borel set B is non-polar if and only if there exists a bounded measure whose support is in $B$ with finite $\lambda$-energy for $X$.

The next lemmas show some peculiarity for sets which are non-polar but semipolar.

Lemma 2.4 (Kanda [6], Rao [11]). Let $K$ be a compact set such that $K \subset\left\{x ; E^{x}\left(\exp \left(-\lambda T_{K}\right)\right)<\delta\right\}$ for some $\delta<1$. Then $C^{\lambda}(K) \uparrow C$ as $\lambda \uparrow \infty$ for some finite constant $C$.

Lemma 2.5 (Kanda [8], Fitzsimmons [3]). Let $K$ be a closed set such that $K \subset\left\{x ; E^{x}\left(\exp \left(-\lambda T_{K}\right)\right) \leq \delta, \hat{E}^{x}\left(\exp \left(-\lambda \hat{T}_{K}\right)\right) \leq \delta\right\}$ for some $\delta<1$. Then $a$ subset $B$ of $K$ is polar if and only if $\pi(B)=0$, where $\pi$ is the $\lambda$-capacitary 
measure of $K$ for $X$, that is, the uniquely determined measure on $K$ such that $\tilde{U}^{\lambda} * \pi(x)=E^{x}\left(\exp \left(-\lambda T_{K}\right)\right)$.

In the above we used the dual process of $X$ with the symbol " $\wedge$ " attached. But recently Fitzsimmons noted that $K \subset\left\{x ; E^{x}\left(\exp \left(-\lambda T_{K}\right)\right) \leq \delta\right\}$ is sufficient for the statement [3].

The following lemma gives a relation between a measure which does not charge semipolar sets and its energy.

Lemma 2.6 (Rao [12], Kanda [7]). If $\nu$ is a bounded measure which charges no semipolar sets and $E_{X}^{\lambda}(\nu)<\infty$, then $E_{X}^{\lambda}(\nu) \downarrow 0$ as $\lambda \uparrow \infty$.

Finally we give a lemma which is essential in the proof of (II) of Theorem.

Lemma 2.7 (Zabczyk [14]). Let $U$ be a real function on $R^{d}$ of class $L^{1}$. Then there exists a singular measure $\eta$ (relative to the Lebesgue measure) such that $U * \eta$ equals a continuous function on $R^{d}$ except on a set of Lebesgue measure 0.

\section{§ 3. Proof of Theorem (I)}

In the subsequent sections, the process $X$ is a Lévy process on $R^{d}$ with the exponent $\Psi$ which has a transition probability density. Hence the space-time process $Y$ over $X$ is a Lévy process on $R^{1} \times R^{d}$ with the $\lambda$-resolvent density $W^{\lambda}(t, x)$ as is explained in $\S 1$. We denote by $\mathscr{F}$ the Fourier transform on $R^{1} \times K^{d}$. We add the suffixes $x$ and $t$ for the Fourier transforms on the variable $x$ of $R^{d}$ and on the variable $t$ of $R^{1}$, respectively. Thus

$$
\begin{aligned}
& \mathscr{F}_{x}\left(U_{S}^{\lambda} * \nu * \tilde{\nu}\right)(z)=\operatorname{Re}\left([\lambda+\Psi(z)]^{-1}\left|\mathscr{F}_{x} \nu(z)\right|^{2},\right. \\
& \mathscr{F}\left(W_{S}^{\lambda} * \mu * \mu\right)(t, z)=\operatorname{Re}\left([\lambda+\Psi(z)-i t]^{-1}\right)|\mathscr{F} \mu(t, s)|^{2} .
\end{aligned}
$$

In what follows, we assume for simplicity that

$$
\mu \text { is a probability measure on } R^{1} \times R^{d} \text {. }
$$

Then $\mu$ is disintegrated as

$$
\mu(d s d x)=\mu_{2}(d x) \mu_{1}(d s, x),
$$

where $\mu_{2}(d x)\left(=\mu\left(R^{1} \times d x\right)\right.$, the $R^{d}$-marginal of $\left.\mu\right)$ and $\mu_{1}(d s, x)$ are probability measures on $R^{d}$ and $R^{1}$, respectively. 
Proof of i) of the part (I). Set

$$
f(t, x)=\mathscr{F}_{t}\left(\mu_{1}(\circ, x)\right)(t) .
$$

Then $\mathscr{F}(\mu)(t, z)=\mathscr{F}_{x}\left(f(t, x) \mu_{2}(d x)\right)(z)$. By the assumption, the $\lambda$-energy of $\mu$ for $Y$ is finite. So $\int \operatorname{Re}\left([\lambda+\Psi(z)-i t]^{-1}\right)|\mathscr{F}(\mu)(t, z)|^{2} d z<\infty$ for almost all $t$. Since $E_{X}^{\lambda}\left(f(t, x) \mu_{2}(d x)\right)=\int \operatorname{Re}\left([\lambda+\Psi(z)]^{-1}\right)|\mathscr{F}(\mu)(t, z)|^{2} d z$, it follows from the estimate $\operatorname{Re}\left([\lambda+\Psi(z)-i t]^{-1}\right) \geq C \operatorname{Re}\left([\lambda+\Psi(z)]^{-1}\right)$ for every $z$, where $C$ is a positive constant (independent of $z$ but dependent on $t$ ), that $E_{X}^{\lambda}\left(f(t, x) \mu_{2}(d x)\right)<\infty$ for almost all $t$. But

$$
\left|\mathscr{F}_{k}\left(f(t, x) \mu_{2}(d x)\right)(z)\right|^{2}=G_{1}(t, z)+G_{2}(t, z),
$$

where $G_{1}(t, z)=\left|\mathscr{F}_{x}\left(\operatorname{Re} f(t, x) \mu_{2}(d x)\right)(z)\right|^{2}+\left|\mathscr{F}_{x}\left(\operatorname{Im} f(t, x) \mu_{2}(d x)\right)(z)\right|^{2}$ and

$$
\begin{aligned}
G_{2}(t, z)= & 2 \int \cos \langle z, x\rangle \operatorname{Im} f(t, x) \mu_{2}(d x) \int \sin \langle z, x\rangle \operatorname{Re} f(t, x) \mu_{2}(d x) \\
& -2 \int \cos \langle z, x\rangle \operatorname{Re} f(t, x) \mu_{2}(d x) \int \sin \langle z, x\rangle \operatorname{Im} f(t, x) \mu_{2}(d x) .
\end{aligned}
$$

Since $\operatorname{Re}\left([\lambda+\Psi(z)]^{-1}\right)=\operatorname{Re}\left([\lambda+\Psi(-z)]^{-1}\right), G_{1}(t, z)=G_{1}(t,-z)$ and $G_{2}(t, z)$ $=-G_{2}(t,-z)$, we have

$$
\begin{aligned}
\int_{|z|>R} & \operatorname{Re}\left([\lambda+\Psi(z)]^{-1}\right) G_{1}(t, z) d z \\
= & \int_{|z|<R} \operatorname{Re}\left([\lambda+\Psi(z)]^{-1}\right)\left[G_{1}(t, z)+G_{2}(t, z)\right] d z \leq E_{X}^{\lambda}\left(f(t, x) \mu_{2}(d x)\right)<\infty
\end{aligned}
$$

for every $R$. Thus $E_{X}^{\lambda}\left(\operatorname{Re} f(t, x) \mu_{2}(d x)\right)<\infty$. Now note that, by compactness of the support of the measure $\mu$, there exist constants $c>0$ and $\varepsilon>0$ such that $\operatorname{Re} f(t, x)>c$ for every $|t|<\varepsilon$ and every $x$. Hence, using Corollary of Lemma 2.1, we see $E_{X}^{\lambda}\left(\mu_{2}\right)<\infty$. The proof of i) is finished.

Proof of ii) of the part (I). Assume that the $R^{1}$-marginal $\mu_{1}$ of $\mu$ is singular to the Lebesgue measure (we choose a set $E$ of Lebesgue measure 0 such that $\left.\mu_{1}\left(R^{1}-E\right)=0\right)$. Suppose that $R^{d}$-marginal $\mu_{2}$ of $\mu$ charges a semipolar set. Then there exist a constant $\delta, 0<\delta<1$, and a compact set $B$ such that $B \subset\left\{x ; E^{x}\left(\exp \left(-\lambda T_{B}\right) \leq \delta, \hat{E}^{x}\left(\exp \left(\exp \left(-\lambda \hat{T}_{B}\right) \leq \delta\right\}\right.\right.\right.$ and $\mu_{2}(B)>0$. Note that $B$ is non-polar for $X$. Indeed, for the restriction $\left.\mu_{2}\right|_{B}$ of $\mu_{2}$ to the set $B, E_{X}^{\lambda}\left(\left.\mu_{2}\right|_{B}\right)<\infty$ by $E_{X}^{\lambda}\left(\mu_{2}\right)<\infty$ and by Corollary of Lemma 2.1. So $B$ must be non-polar by Lemma 2.3. Let $\pi_{B}$ be the $\lambda$ capacitary measure of the set $B$ for $X$. Then $d t \otimes \pi_{B}$ is the $\lambda$-capacitary measure of the set $R^{1} \times B$ for the space-time process $Y$ over $X$. Indeed, 


$$
\begin{aligned}
\iint W^{\lambda}(t-s, y-x) d t \pi_{B}(d y) & =\int U^{\lambda}(y-x) \pi_{B}(d y) \\
& =E^{x}\left(\exp \left(-\lambda T_{B}\right)\right) \\
& =E^{t, x}\left(\exp \left(-\lambda T_{R^{1} \times B}\right)\right)
\end{aligned}
$$

where $T_{R 1 \times B}=\inf \left(t>0, Y_{t} \in R^{1} \times B\right)$. Clearly $\left(d t \otimes \pi_{B}\right)(E \times B)=0$. So, applying Lemma 2.5 for $Y$, the set $E \times B$ must be polar for $Y$. But, disintegrating $\mu$ as $\mu_{1}(d s) \mu_{2}(s, d x)$,

$$
\begin{aligned}
\mu(E \times B) & =\iint_{E \times B} \mu_{1}(d s) \mu_{2}(s, d x) \\
& =\iint_{R^{1 \times B}} \mu_{1}(d s) \mu_{2}(s, d x)=\mu\left(R^{1} \times B\right)=\mu_{2}(B)>0 .
\end{aligned}
$$

Since the $\lambda$-energy of $\mu$ for $Y$ is finite by the assumption, the set $E \times B$ must be non-polar for $Y$ by Lemma 2.3. Thus the $R^{d}$-marginal $\mu_{2}$ does not charge a semipolar set. The proof of ii) is finished.

\section{§4. Proof of Theorem (II)}

We use the same symbols as in $\S 3$. In the case of $\mu=\eta \otimes \nu, \mu_{1}(d t)$ $=\eta(d t)=\mu_{1}(d t, x), \mu_{2}(d x)=\nu(d x)=\mu_{2}(t, d x)$ and so

$$
E_{X}^{\lambda}(\mu)=\iint \operatorname{Re}\left([\lambda+\Psi(z)-i t]^{-1}\right)\left|\mathscr{F}_{t} \eta(t)\right|^{2}\left|\mathscr{F}_{x} \nu(z)\right|^{2} d t d z .
$$

Proof of i) of the part (II). First note that $E_{X}^{\lambda}(\nu)<\infty$ follows from $E_{Y}^{\lambda}(\mu)<\infty$ by i) of (I). If $\nu$ charges a semipolar set, then charges a compact set $K$ such that $K \subset\left\{x ; E^{x}\left(\exp \left(-\lambda T_{K}\right)\right)<\delta\right\}$ for some $\delta<1$. Let $\nu_{K}$ be the restriction of $\nu$ to the set $K$. Then $E_{X}^{\lambda}\left(\nu_{K}\right) \leq E_{X}^{\lambda}(\nu)<\infty$ by Corollary of Lemma 2.1, and therefore $K$ must be non-polar for $X$ by Lemma 2.3. So $C^{\lambda}(K) \uparrow C$ as $\lambda \uparrow \infty$ for some positive finite constant $C$ by Lemma 2.4. Then it follows from Lemma 2.2 that

$$
\lim _{\lambda \uparrow \infty} E_{X}^{\lambda}(\nu) \geq \lim _{\lambda \uparrow \infty} E_{X}^{\lambda}\left(\nu_{K}\right) \geq(2 \pi)^{d} \nu(K)^{2} / 2 C .
$$

Thus we have

$$
\liminf _{\lambda \uparrow \infty} \int \operatorname{Re}\left([\lambda+\Psi(z)-i t]^{-1}\right)|\mathscr{F} \nu(z)|^{2} d z \geq(2 \pi)^{d} \nu(K)^{2} / 2 C
$$

for every fixed $t$. Hence

$$
\lim _{\lambda \uparrow \infty} E_{Y}^{\lambda}(\mu) \geq \int\left|\mathscr{F}_{t} \eta(t)\right|^{2} d t(2 \pi)^{d} \nu(K)^{2} / 2 C .
$$


So $\mathscr{F}{ }_{t} \eta$ belongs to $L^{2}\left(R^{1}\right)$, which implies that $\eta$ is absolutely continuous and that the density belongs to $L^{2}\left(R^{1}\right)$. The proof of i) of the part (II) is finished.

Proof of ii) of the part (II). Let $\nu$ be a bounded measure with finite $\lambda$-energy for $X$. Assume that the measure $\nu$ does not charge any semipolar set. Then, by Lemma 2.6,

$$
E_{X}^{\lambda}(\nu) \downarrow 0 \quad \text { as } \quad \lambda \uparrow \infty .
$$

Set

$$
g_{\lambda}(t, x)=\int W_{S}^{\lambda}(t, y-x) \nu * \tilde{\nu}(d y)
$$

Then

$$
\int_{-\infty}^{\infty} g_{\lambda}(t, x) d t=U_{S}^{\lambda} * \nu * \tilde{\nu}(x) .
$$

Since $U_{S}^{\lambda} * \nu * \tilde{\nu}$ is bounded by Lemma $2.1, g_{\lambda}(t, 0)$ is $L^{1}$ in $t$. So it follows from Lemma 2.7 that there exists a bounded singular measure $\eta$ on $R^{1}$ (we may suppose its support is compact) such that $g_{2}(\cdot, 0) *_{(\ell)} \eta$ equals a continuous function on $R^{1}$, a.e., and therefore $g_{2}(\cdot, 0) *_{(t)} \eta$ is locally bounded because of its lower semicontinuity. Hence $g_{\lambda}(\cdot, 0) *_{(t)} \eta *_{(t)} \tilde{\eta}$ is locally bounded in $t$. Clearly it belongs to $L^{1}\left(R^{1}\right)$. Further, for every $t$,

$$
\mathscr{F}_{t}\left(g_{\lambda}(\cdot, 0)\right)(t)=\left[\mathscr{F}_{t}\left(W_{S}^{\lambda}(\cdot, x)\right)(t) *_{(x)} \nu *_{(x)} \tilde{\nu}\right](0)
$$

by Fubuni's theorem. (In the above we denote by $*_{(t)}$ and $*_{(x)}$ the convolution operation in $t$ and $x$ respectively.) On the other hand, since

$$
\mathscr{F}\left(W_{S}^{\lambda}\right)(t, z)=\mathscr{F}_{x}\left[\mathscr{F}_{t}\left(W_{S}^{\lambda}(\cdot, x)\right)(t)\right](z)=\operatorname{Re}\left([\lambda+\Psi(z)-i t]^{-1}\right),
$$

we have, for each fixed $t$,

$$
\mathscr{F}_{x}\left[\mathscr{F}_{t}\left(W_{S}^{\lambda}(\cdot, x)\right)(t) *_{(x)} \nu *_{(x)} \tilde{\nu}\right](z)=\operatorname{Re}\left([\lambda+\Psi(z)-i t]^{-1}\right)\left|\mathscr{F}_{x} \nu(z)\right|^{2} \geq 0 .
$$

Hence it follows from Bochner's theorem that, for each fixed $t$,

$$
\begin{aligned}
& \left(\mathscr{F}_{t}\left(W_{S}^{\lambda}(\cdot, \cdot)\right)(t) *_{(x)} \nu *_{(x)} \tilde{\nu}\right)(x) \\
& \quad=\mathscr{F}_{x}^{-1}\left[\operatorname{Re}\left([\lambda+\Psi(\cdot)-i t]^{-1}\right)\left|\mathscr{F}_{x} \nu(\cdot)\right|^{2}\right](x)
\end{aligned}
$$

for almost all $x$. In general the equality does not hold for all $x$. In the following we shall show the equality holds for $x=0$ (hence it holds everywhere) by the use of (4.1). Since $\mathscr{F}_{t}\left(g_{\lambda}(\cdot, 0)\right)(t)=\left(\mathscr{F}_{t}\left(W_{S}^{\lambda}(\cdot, \cdot)\right)(t)\right.$ $\left.*_{(x)} \nu *_{(x)} \tilde{)}\right)(0)$, we must show 


$$
\mathscr{F}_{t}\left(g_{\lambda}(\cdot, 0)\right)(t)=(2 \pi)^{-d} \int \operatorname{Re}\left([\lambda+\Psi(z)-i t]^{-1}\right)\left|\mathscr{F}_{x^{\nu}}(z)\right|^{2} d z
$$

Define

$$
V_{t}^{\lambda}(x)=\int \exp (i t u) W^{\lambda}(u, x) d u / 2, \quad \hat{V}_{t}^{\lambda}(x)=\int \exp (i t u) W^{\lambda}(-u,-x) d u / 2 .
$$

Then it is easily proved that

$$
V_{t}^{\lambda}(z)-V_{t}^{\lambda^{\prime}}(z)=2\left(\lambda^{\prime}-\lambda\right) \int V_{t}^{\lambda}(y) V_{t}^{\lambda^{\prime}}(z-y) d y .
$$

The same equality is also valid for $\hat{V}_{t}^{\lambda}$. Setting $H^{2}(t, z)=\left(\left(V_{t}^{\lambda}+\hat{V}_{t}^{\lambda}\right) * \nu * \tilde{\nu}\right)(z)$, we have

$$
\begin{aligned}
H^{\lambda}(t, z)-H^{\lambda^{\prime}}(t, z)= & 2\left(\lambda^{\prime}-\lambda\right) \int V_{t}^{\lambda}(x+z)\left[\int V_{t}^{\lambda^{\prime}}(y-x) \nu * \tilde{\nu}(d y)\right] d x \\
& +2\left(\lambda^{\prime}-\lambda\right) \int \hat{V}_{t}^{\lambda}(x+z)\left[\int \hat{V}_{t}^{\lambda^{\prime}}(y-x) \nu * \tilde{\nu}(d y)\right] d x .
\end{aligned}
$$

Since $\int V_{t}^{\lambda}(y-x) \nu * \tilde{\nu}(d y)$ and $\int \hat{V}_{t}^{\lambda^{\prime}}(y-x) \nu * \tilde{\nu}(d y)$ are bounded measurable, each term of the right side is a continuous function of $z$, and so $H^{\lambda}(t, z)-H^{\lambda^{\prime}}(t, z)$ is continuous. Since $H^{\lambda}(t, z)=\left(\mathscr{F}_{t}\left(W_{S}^{\lambda}(\cdot, x)\right)(t) *_{(x)} \nu *_{(x)} \tilde{\nu}\right)(z)$, it follows from (4.2) that

$$
\begin{gathered}
\left(\mathscr{F}_{t}\left(W_{S}^{\lambda}(\cdot, x)\right)(t) *_{(x)} \nu *_{(x)} \tilde{\nu}\right)(z)-\left(\mathscr{F}_{t}\left(W_{S}^{\lambda^{\prime}}(\cdot, x)\right)(t) *_{(x)} \nu *_{(x)} \tilde{\nu}\right)(z) \\
=\mathscr{F}_{x}^{-1}\left[\operatorname{Re}\left([\lambda+\Psi(\cdot)-i t]^{-1}\right)\left|\mathscr{F}_{x} \nu(\cdot)\right|^{2}\right](z) \\
-\mathscr{F}_{x}^{-1}\left[\operatorname{Re}\left(\left[\lambda^{\prime}+\Psi(\cdot)-i t\right]^{-1}\right)\left|\mathscr{F}_{x^{\prime}} \nu(\cdot)\right|^{2}\right](z)
\end{gathered}
$$

for every $z$. In particular, putting $z=0$ and letting $\lambda^{\prime} \uparrow \infty$, we have

$$
\begin{aligned}
\mathscr{F}_{t}\left(g_{\lambda}(\cdot, 0)\right)(t)= & (2 \pi)^{-d} \int \operatorname{Re}\left[(\lambda+\Psi(z)-i t]^{-1}\right)\left|\mathscr{F}_{x^{\nu}} \nu(z)\right|^{2} d z \\
& -\lim _{\lambda^{\prime} \uparrow \infty}(2 \pi)^{-d} \int \operatorname{Re}\left(\left[\lambda^{\prime}+\Psi(z)-i t\right]^{-1}\right)\left|\mathscr{F}_{x^{\nu}} \nu(z)\right|^{2} d z .
\end{aligned}
$$

But it follows from (4.1) that the last term in the above equality is zero. Thus the equality (4.3) is proved. Finally we shall prove that the $\lambda$ energy of $\mu=\eta \otimes \nu$ for $Y$ is finite. Since

$$
\begin{aligned}
\mathscr{F}_{t}\left(g(\cdot, 0) *_{(t)} \eta *_{(t)} \tilde{\eta}\right)(t)=\mathscr{F}_{t}\left(g_{\lambda}(\cdot, 0)\right)(t)\left|\mathscr{F}_{t} \eta(t)\right|^{2} \\
=(2 \pi)^{-d} \int \operatorname{Re}\left([\lambda+\Psi(z)-i t]^{-1}\right)\left|\mathscr{F}_{x} \nu(z)\right|^{2} d z\left|\mathscr{F}_{t} \eta(t)\right|^{2} \geq 0
\end{aligned}
$$

by (4.3), Bochner's theorem ensures that 


$$
\int \operatorname{Re}\left([\lambda+\Psi(z)-i t]^{-1}\right)\left|\mathscr{F}_{x} \nu(z)\right|^{2} d z\left|\mathscr{F}_{t} \eta(t)\right|^{2}
$$

belongs to $L^{1}\left(R^{1}\right)$ as a function of $t$, which implies $E_{Y}^{\lambda}(\eta \otimes \nu)<\infty$. The proof of ii) of the part (II) is now finished.

\section{$\S 5 . \quad$ Proof of Corollary}

First we shall prove the "only if" part. Assume that the set $B$ is semipolar for $X$. If $B$ is polar, the assertion is trivial. So we assume that $B$ is non-polar. If there exists a set $A$ in $] 0, \infty$ [ of Lebesgue measure zero such that $P^{x}\left(X_{t} \in B\right.$ for some $\left.t \in A\right)>0$ for some $x$. Then the product set $A \times B$ in $R^{1} \times R^{d}$ is non-polar for the space-time process $Y$ over $X$. So there exists a bounded measure $\mu$ whose support is compact and in $A \times B$ with finite $\lambda$-energy for $Y$ by Lemma 2.3. Then the $R^{1}$-marginal $\mu_{1}$ of $\mu$ is carried by $A$ and the $R^{d}$-marginal $\mu_{2}$ of $\mu$ is carried by $B$. This contradicts the statement ii) of the part (I) in Theorem.

Before proving the "if" part, we prepare

LEMMA 5.1. Let $B$ be a non-semipolar closed set. Then there exists $a$ non-trivial bounded measure $\nu$ on $B$ of compact support with finite $\lambda$-energy for $X$ that charges no semipolar set. Indeed we can choose the restriction of the regular part (explained below) of the $\lambda$-capacitary measure of $B$ for $X$ to some compact subset of $B$ as the measure $\nu$.

Proof. We can decompose any bounded measure $\mu$ as $\mu=\mu_{1}+\mu_{2}+\mu_{3}$ where $\mu_{1}$ is carried by a polar Borel set, $\mu_{2}$ is carried by a semipolar Borel set but charges no polar set and $\mu_{3}$ charges no semipolar set. See Blumenthal and Getoor [1], p. 283. We say that $\mu_{3}$ is the regular part of $\mu$. We show that the regular part of the $\lambda$-capacitary measure $\pi_{B}$ of $B$ for $X$ is non-trivial (i.e. $\left(\pi_{B}\right)_{3} \neq 0$ ). Suppose, on the contrary, that the regular part is trivial. Since $\pi_{B}$ charges no polar set, we have then $\pi_{B}$ $=\left(\pi_{B}\right)_{2}$. Let $E$ be a semipolar Borel subset of $B$ for $X$ such that $\pi_{B}(B-E)$ $=0$. Then $E$ is a countable union of thin sets for $X$ by definition. Let $H$ be any compact subset of one of such thin sets satisfying $\pi_{B}(H)>0$. Let $\mu$ and $\nu$ be the restrictions of $\pi_{B}$ to $B$ and $B-H$, respectively. Then $U^{2} \mu$ is discontinuous at $\mu$-almost all points by Pop-Stojanovic [10]. But $E^{x}\left(\exp \left(-\lambda T_{B}\right)\right)=\tilde{U}^{\lambda} * \pi_{B}(x)=\tilde{U}^{\lambda} * \mu(x)+\tilde{U} * \nu(x)$, and so $E^{x}\left(\exp \left(-\lambda T_{B}\right)\right)$ is continuous at $x$ if and only if both $\tilde{U}^{\lambda} * \mu$ and $\tilde{U}^{\lambda} * \nu$ are continuous at $x$, because the both are lower-semicontinuous. Since $E^{x}\left(\exp \left(-\lambda T_{B}\right)\right)$ 
is continuous at every point of $B^{r}\left(=\left\{x ; E^{x}\left(\exp \left(-\lambda T_{B}\right)\right)=1\right\}\right)$, we see $\mu\left(B^{r}\right)=0$. Therefore $\pi_{B}\left(B^{r}\right)=0$, because $\pi_{B}\left(B^{r} \cap H\right)=\mu\left(B^{r}\right)=0$ for every $H$ and so $0=\pi_{B}\left(B^{r} \cap E\right)=\pi_{B}\left(B^{r} \cap B\right)=\pi_{B}\left(B^{r}\right)$. For the last equality we used the closedness of $B$. Setting $D=B-B^{r}$, we have then $\left.\pi_{B}\right|_{D}$ (= the restriction of $\pi_{B}$ to $\left.D\right) \leq \pi_{D}$, where $\pi_{D}$ is the $\lambda$-capacitary measure of $D$ for $X$, because

$$
\begin{aligned}
\pi_{B}(S) & =\lambda \int \hat{E}^{x}\left(\exp \left(-\lambda \hat{T}_{B}\right), \hat{X}_{\hat{T}_{B}} \in S\right) d x \leq \lambda \int \hat{E}^{x}\left(\exp \left(-\lambda \hat{T}_{D}\right), \hat{X}_{\hat{T}_{D}} \in S\right) d x \\
& =\pi_{D}(S)
\end{aligned}
$$

for $S \subset D$. So $E^{x}\left(\exp \left(-\lambda T_{B}\right)\right)=\left.\tilde{U}^{\lambda} * \pi_{B}\right|_{D}(x) \leq \tilde{U}^{\lambda} * \pi_{D}(x)=E^{x}\left(\exp \left(-\lambda T_{D}\right)\right)$. Since $T_{D} \geq T_{B}$ almost surely, we have $P^{x}\left(T_{B}=T_{D}\right)=1$ for every $x$. But the set $D$ is semipolar so that almost surely $X_{t} \in D$ for only countable many values of $t$. See Blumenthal and Getoor [1], p. 80. Then it follows from $D=B-B^{r}$ and $T_{B}=T_{D}$ almost surely that $X_{t} \in B$ for only countably many values of $t$ almost surely. Hence the set $B$ must be semipolar. See Sharpe [13], p. 281. This contradicts the assumption that $B$ is nonsemipolar.

Now we prove the "if" part of Corollary. Assume that $B$ is nonsemipolar for $X$. Then there exists a bounded measure $\nu$ on $B$ of compact support with finite $\lambda$-energy for $X$ which charges no semipolar set. For the measure $\nu$, by ii) of the part (II) in Theorem, we can find a singular measure $\eta$ on $R^{1}$ such that $\eta \otimes \nu$ has finite $\lambda$-energy for $Y$. Then the product set $E \times B$ is non-polar for $Y$ by Lemma 2.3, where $E$ is a set of Lebesgue measure zero such that $\eta\left(R^{1}-E\right)=0$. This implies $P^{x}\left(X_{t} \in B\right.$ for some $\left.t \in A\right)>0$ for some $x$ and for some set $\left.A \subset\right] 0, \infty[$ of Lebesgue measure zero (which is indeed a translation of $E$ ). The proof of Corollary is finished.

Remark. If the process $X$ satisfies Hunt's condition $(\mathrm{H})$, that is, every semipolar set for $X$ is polar for $X$, then $a$ set $B$ is polar if and only if $P^{x}\left(X_{t} \in B\right.$ for some $\left.t \in A\right)=0$ for every $x$ and every set $\left.A \subset\right] 0, \infty[$ of Lebesgue measure zero.

Acknowledgement. The author is grateful to the referee for his many corrections and remarks. 


\section{REFERENCES}

[1] R. G. Blumenthal and R. K. Getoor, Markov Processes and Potential Theory, Academic Press, New York, 1968.

[2] S. Bochner, Harmonic Analysis and Theory of Probability, Berkeley: Univ. of California Press 1955.

[ 3 ] P. J. Fitzsimmons, Remarks on a paper of Kanda, (private communication), (1989).

[4] J. Hawkes, Potential theory of Lévy processes, Proc. London Math. Soc., (3) 38 (1979), 335-352.

[5] M. Kanda, Two theorems on capacity for Markov processes with stationary independent increments, Z. Wahrsch. Verw. Gebiete, 35 (1976), 159-165.

[6] - Characterizations of semipolar sets for processes with stationary independent increments, Z. Wahrsch. Verw. Gebiete, 42 (1978), 141-154.

[ 7 ] — Characterization of semipolar sets for Lévy processes by Fourier transform of measures, Surikaiseki Kenkyusho Kokyuroku, 502 (1983), 161-170.

[ 8 ] _ - A note on capacitary measures of semipolar sets, The Annals of Probability, (1) 17 (1989), 379-384.

[ 9 ] — A note on a characterization of semipolar sets, Abstracts, Singapore Prob. Conference (1989).

[10] Z. R. Pop-Stojanovic, Energy in Markov processes, Functional analysis, (II) (Dubrovnik 1985), Lecture Notes in Math., 1242 (1987), 374-395.

[11] M. Rao, On polar sets for Lévy processes, J. London Math. Soc., (2) 35 (1987), $569-576$.

[12] - Hunt's hypothesis for Lévy processes, Proc. Amer. Math. Soc., (2) 104 (1988), 621-624.

[13] M. Sharpe, General Theory of Markov Processes, Academic Press, San Diego, 1988.

[14] J. Zabczyk, A note on semipolar sets for processes with independent increments, Winter School on Probability, Karpacz, Poland, Lecture Notes in Math., 472 (1975) 277-283.

Department of Mathematics

University of Tsukuba

Tsukuba-shi, Ibaraki 305

Japan 